\title{
Die distale Radiusfraktur: Behandlungsstrategie beim Erwachsenen
}

\author{
Karl Heinrich Winker, Daniel Rikli
}

\section{Zusammenfassung}

Bei Nachuntersuchungen festgestellte mangelhafte Ergebnisse nach jahrzehntelang durchgeführter konservativer Behandlung der distalen Radiusfraktur beim Erwachsenen führte Mitte der 1990er-Jahre zu einem Therapiewandel: Die operative Behandlung zunächst durch Osteosynthesen mit Kirschner-Drähten, mit dem Fixateur externe oder mit von palmar oder dorsal angewendeten konventionellen 3,5-mm-Platten wurde ergänzt durch winkelstabile Implantate. Mittlerweile sind diese 2,4- bis 3,5-mm-Plattensysteme in ihrer Anwendung von überwiegend palmar und bei grob dislozierten intraartikulären Frakturen (C2, C3) auch von dorsal zum Standard geworden. Bei Beachtung der korrekten Indikation, technischen Durchführung und Nachbehandlung ist mit nur noch niedrigen Komplikationsraten zu rechnen.

\section{Treatment Strategies for Adults}

Unsatisfactory results detected following conservative treatment of distal radius fractures have led to a change of paradigm: during the mid-nineties of the last century operative procedures like percutaneous K-wiring, external fixation and plating with conventional $3.5-\mathrm{mm}$ plate systems were supplemented by the invention of locking plates. Meanwhile palmar plating of even extension fractures with angular stable plate systems of the $2.4-3.5 \mathrm{~mm}$ group have become a standard treatment. Only wide displaced intraarticular extension fractures of type C2 or C3 need to be addressed from dorsally. With consideration of the correct indications, operative technique and rehabilitation only low complication rates are to be expected.

\section{Therapieziel}

Hauptziel der Behandlung ist die anatomische und funktionelle Wiederherstellung der Hand unter Berücksichtigung des Frakturtyps und evtl. Begleitverletzungen $[8,9]$. Operiert werden die per se instabilen Flexionsfrakturen sowie instabile Extensionsfrakturen, wenn hier mindestens 2 von 6 Instabilitätskriterien vorliegen [14] (Tab. 1). Durch die OP sollen die Böhler-Winkel, die radiale Länge sowie die stufenfreie Gelenkfläche wiederhergestellt und ligamentäre Begleitverletzungen mitversorgt werden [9].

OP-JOURNAL 2012; 28: 252-255

(c) Georg Thieme Verlag KG Stuttgart · New York DOI http://dx.doi.org/10.1055/s-0032-1328050

\section{OP-Zeitpunkt}

Alle grob dislozierten oder Luxationsfrakturen werden unter Anästhesie schnellstmöglich reponiert und dann zeitnah operiert $[6,12]$. Offene Brüche oder Störungen des N. medianus müssen notfallmäßig operiert werden.

\section{OP-Verfahren}

\section{Osteosynthese mit Kirschner-Drähten/} kanülierten Schrauben $[4,14]$

Bei guter Knochenqualität können mit diesem Verfahren stabile A2-, instabile A3-, B1- und C1-Frakturen versorgt werden. Nach Armtischlagerung wird unter Anästhesie geschlossen unter Bildwandlerkontrolle reponiert und über kleine Hautinzisionen über dem Processus styloideus radii unter Schutz einer Bohr- hülse 1,6- oder 1,8-mm-Kirschner-Drähte bzw. eine kanülierte 2,7- oder 3,5mm-Schraube eingebracht. Man unterscheidet eine intrafokale Drahtplatzierung nach Kapandji von einer extrafokalen Drahtplatzierung nach Willenegger. Die Drähte werden umgebogen und wie Schrauben subkutan versenkt. Zur Nachbehandlung ist für meist 4 Wochen eine Ruhigstellung im Fixateur externe, im Gips oder in einer Orthese erforderlich. Drähte können nach 6 Wochen entfernt werden, Schrauben nur bei Bedarf. Bei korrekter Indikation und technischer Durchführung können gute Ausheilungsergebnisse erzielt werden. Nachteilig ist die Notwendigkeit der Drahtentfernung, das Risiko einer sekundären Dislokation oder Implantatlockerung, evtl. Infektion, insbesondere bei schlechter Knochenqualität. Insofern kann die Osteosynthese mit Kirschner-Drähten bei alten Patienten nur ausnahmsweise empfohlen werden.

Die Osteosynthese mit Kirschner-Drähten kann bei alten Patienten nur noch ausnahmsweise empfohlen werden

\section{Fixateur externe}

Bei polytraumatisierten Patienten und/ oder Frakturen nach Hochenergietrauma mit höhergradig geschlossenem oder offenem Weichteilschaden ist die meist temporäre Ruhigstellung von A3-Frakturen, aber insbesondere von komplexen

\begin{tabular}{l}
$\begin{array}{l}\text { Tab. } 1 \text { Instabilitätskriterien bei distalen } \\
\text { Radiusextensionsfrakturen nach Jupiter. }\end{array}$ \\
$\begin{array}{l}\text { - Verkippung der radialen Gelenkfläche } \\
\text { über } 20^{\circ}\end{array}$ \\
\hline - metaphysäre Trümmerzone \\
\hline - axiale Einstauchung über $2 \mathrm{~mm}$ \\
\hline - dislozierte intraartikuläre Fraktur \\
\hline - radioulnare Instabilität \\
\hline - assoziierte distale Ulnafraktur
\end{tabular}




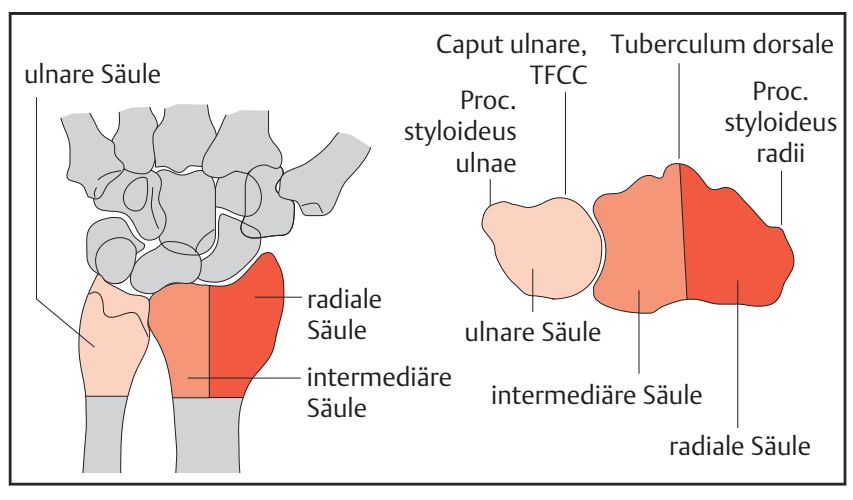

Abb. 1 Drei-SäulenModell.
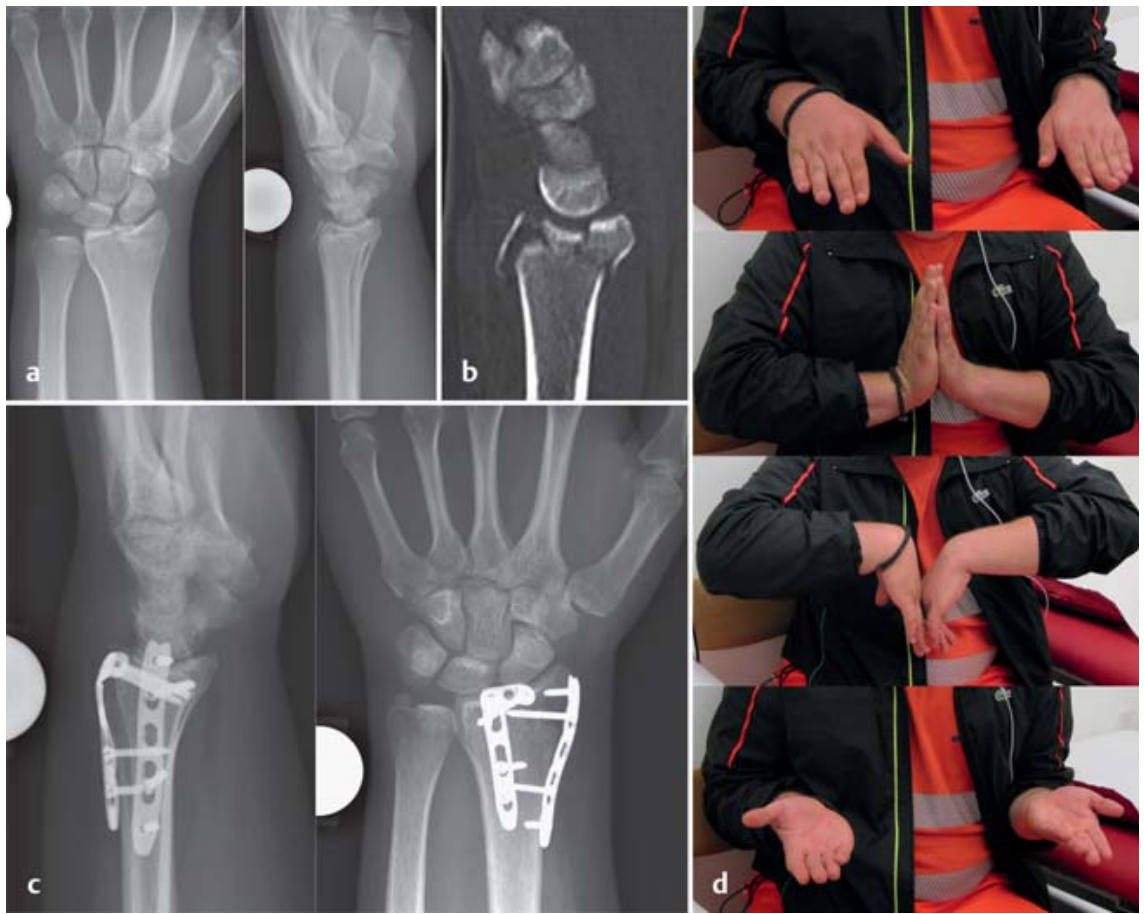

c

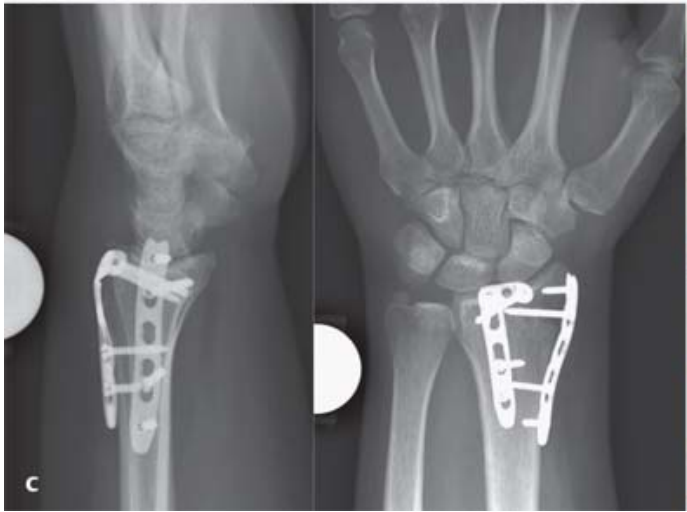

Abb. 2a bis d Plattenosteosynthese von dorsal, Pat. C. B., 23 J., M. a 23-C2, b CT sagittal, c Plattenosteosynthese $(2,4 \mathrm{~mm})$ von dorsal und radial, d Funktion.

intraartikulären Frakturen im Fixateur externe angezeigt [14]. Unter Nutzung des Prinzips der Ligamentotaxis können so gute Repositionsergebnisse erzielt werden. Ein im Fixateur externe durchgeführtes CT erlaubt eine sehr genaue Planung der definitiven Versorgung (3Stufen-Konzept nach Rikli):

1. Reposition und Fixateur externe

2. CT

3. definitive Versorgung

Nach Stichinzisionen über dem MC2 radiodorsal werden 2 Schanz-Schrauben der Stärke $3 \mathrm{~mm}$ nach Vorbohren mit einem 2-mm-Bohrer eingedreht. Gleiches Vorgehen am distalen Radius ca. $10 \mathrm{~cm}$ proximal des Processus styloideus radii. Dieses Vorgehen erlaubt eine spätere Plattenosteosynthese von dorsal oder palmar bei liegendem Fixateur. Spätestens nach etwa 10 Tagen muss zur Schonung der ligamentären Strukturen der Längszug evtl. nachgelassen werden. Im Fixateur externe sollte nicht länger als 6 Wochen behandelt werden, idealerweise erfolgt nach einigen Tagen die offene Reposition und interne Osteosynthese. Wie bei der KD-Osteosynthese besteht das Risiko von Lockerung und/oder Infektion der Schanz-Schrauben, insbesondere bei schlechter Knochenqualität [14].

\section{Plattenosteosynthese nach dem 3-Säulen-Modell (Abb. 1)}

Die plattenosteosynthetische Versorgung distaler Radiusfrakturen sollte gemäß dem 3-Säulen Modell nach folgenden Grundsätzen erfolgen: alle 3 Säulen sollten stabil resp. stabilisiert sein; die radiale Säule kann sowohl von dorsal als auch von palmar abgestützt werden; die intermediäre Säule ist der Schlüssel zum radiokarpalen Gelenk, die formale Revision der Gelenkfläche erfolgt über eine dorsale Arthrotomie; dorsoulnares und palmar-ulnares Fragment sollen individuell angegangen werden; ein hyperextendiertes palmares Fragment sollte von palmar angegangen werden; stellt sich ein disloziertes dorsoulnares Fragment mit Ligamentotaxis nicht ein, wird es von dorsal angegangen. Das Ziel der Osteosynthese ist die stabile Fixation im Hinblick auf eine frühfunktionelle Nachbehandlung. Verwendet werden heute ausschließlich winkelstabile Plattensysteme.

Die Indikation zur dorsalen Plattenosteosynthese hat sich nach Einführung kleinerer Plattensysteme im 2,0- bis 2,7-mm-Format oder in der Doppelplattentechnik mit einem 2,4-mm-System deutlich erweitert $[7,9]$. Dabei wird über einen längs verlaufenden dorsalen Hautschnitt das Retinaculum extensorum freigelegt. Nach z-förmiger Inzision über dem 3. Strecksehnenfach (bedarfsweise auch unter dem 2. oder 4. Strecksehnenfach) wird die Sehne des M. extensor pollicis longus herausgelöst und die Fraktur dargestellt. Größere knöcherne Defekte können mit Spongiosa oder Knochenersatzmaterial aufgefüllt werden. Die frühfunktionelle Nachbehandlung wird angestrebt. Die Anwendung einer konfektionierten Handgelenksorthese für ca. 4 Wochen wird empfohlen ([14], Abb. 4). Probleme können Sehnenirritationen bis hin zu Sehnenrupturen hervorrufen. Nachteilig ist an jeder Form der dorsalen Plattenosteosynthese die Zugangsmorbidität mit der Mobilisierung von Strecksehnen und die meist notwendige Metallentfernung (Abb. 2).

Mit der palmaren Plattenosteosynthese können, insbesondere nach Einführung der winkelstabilen Implantate, nahezu alle instabilen Flexions- und Extensionsfrakturen behandelt werden $[1,3,5,10$, 12-14]. Lediglich bei hochgradig instabilen, intraartikulären Trümmerbrüchen vom Extensionstyp (C3) kann dieses Verfahren überfordert werden und den Einsatz einer evtl. zusätzlichen Versorgung von dorsal notwendig machen. Über den radiopalmaren Zugang zum distalen Radius werden Flexionsfrakturen nach dem Abstützplattenprinzip versorgt. Unter Verwendung von winkelstabilen Plattensystemen werden auch Extensions- 


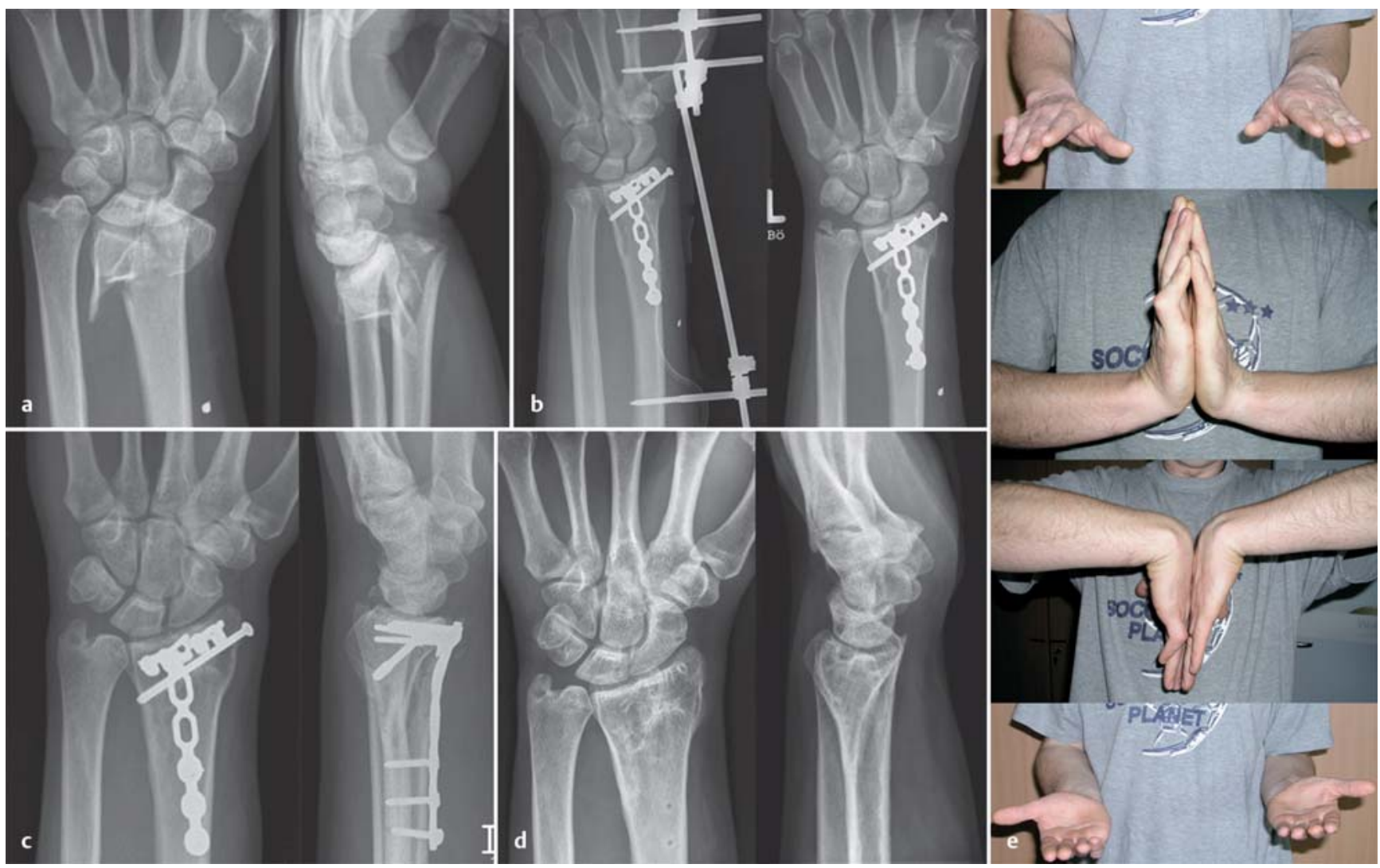

Abb. 3 a bis e Patient K. L., 39]., Leitersturz. C2-Fraktur, palmare winkelstabile Plattenosteosynthese (2,4/2,7 mm). a 23-C2, b FixEx - ORIF, Entfernung FixEx nach 10 Tagen, $\mathbf{c}+2$ Jahre, d ME + 1 J., e Funktion.

frakturen nach dem Prinzip des internen Fixateurs behandelt. Dabei werden die Kräfte aus der subchondralen Schicht der distalen Radiusgelenkfläche von der winkelstabilen Platte aufgenommen und proximal der Fraktur wieder in den Radius eingeleitet. Die Platzierung des Implantats geschieht über einen längs verlaufenden Hautschnitt ulnar der A. radialis. Die Sehne des M. flexor carpi radialis wird dargestellt und nach ulnar gehalten. Nach Spaltung der Unterarmfaszie werden die Beuger ohne Darstellung des $\mathrm{N}$. medianus ebenfalls nach ulnar gehalten und der Pronator quadratus von seinem radialen Ansatz abgetrennt. Die nun freiliegende Fraktur kann über 2 grundsätzlich verschiedene Wege reponiert und stabilisiert werden. Einmal direkt über eine schrittweise Reposition der Fragmente und die abschließende Platzierung der Platte, wobei sich hier Implantate mit multidirektionaler Winkelstabilität bewährt haben. Als 2. Variante kommt eine indirekte Reposition mit der winkelstabilen Platte selbst infrage. Hier werden im 1 . Schritt die winkelstabilen Elemente im Querschenkel der Platte subchondral verankert und dann wird erst durch das Heranführen des Längsschenkels an den Radiusschaft die eigentliche Reposition herbeigeführt. Eine Spongiosaplastik bei knöchernen Defekten ist seit Einführung winkelstabiler Plattensysteme nur ausnahmsweise angezeigt.

Am Ende der palmaren Plattenosteosynthese wird immer häufiger der M. pronator quadratus zu refixieren versucht.

Flexionsfrakturen werden durch palmare Plattenosteosynthesen übungsstabil versorgt und sollten lediglich bis zur gesicherten Wundheilung auf einer volaren Schiene ruhen. Extensionsfrakturen lassen sich unter Verwendung von winkelstabilen Implantaten mit streng subchondral platzierten Kraftträgern ebenfalls übungsstabil versorgen. Auch hier kann nach Konsolidierung der Weichteile mit der frühfunktionellen Beübung begonnen werden. Lediglich bei hochgradig instabilen Frakturen mit Osteoporose sollte für 2-4 Wochen eine zusätzliche Ruhigstellung im Fixateur externe oder Orthese (Abb. 4) erfolgen. Die ME ist nur ausnahmsweise erforderlich. Nach anatomischer Reposition, winkelstabiler Plattenosteosynthese und frühfunktioneller NB werden in der Literatur überwiegend gute und sehr gute Ergeb-

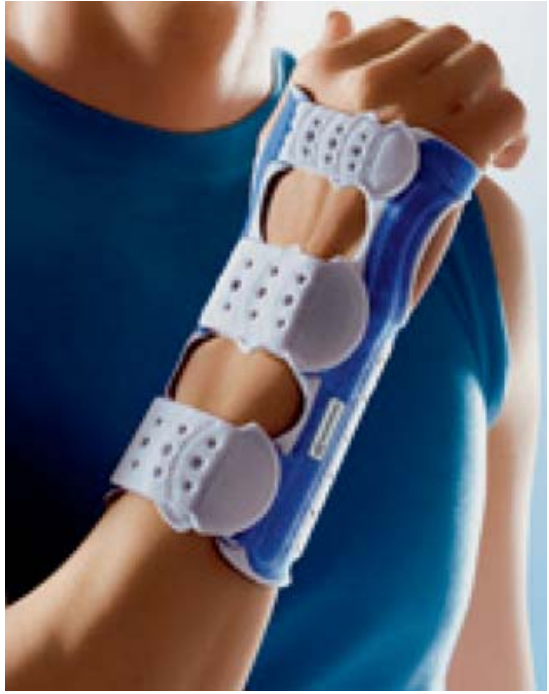

Abb. 4 Konfektionierte Handgelenksorthese (Manoloc - Fa. Bauerfeind).

nisse beschrieben ([6,13,14], Abb.3). Eine typische Komplikation der palmaren Plattenosteosynthese sind Strecksehnenirritationen bei dorsal überstehenden Schraubenspitzen. Das komplexe regionale Schmerzsyndrom (CRPS) wird nach schonender Reposition und frühfunktioneller Beübung nur noch in Einzelfällen angetroffen [12]. 


\section{Marknagel}

Eine relativ neue Entwicklung zur Behandlung von distalen Radiusfrakturen ist die intramedulläre Retention mit einem winkelstabilen Nagel-Platten-Hybridsystem. Es wird gegenwärtig vorzugsweise für instabile extraartikuläre Frakturen (A3) verwendet. Einzelne evaluierte Ergebnisse liegen vor [2].

\section{Spezielle postoperative Komplikationen}

- Strecksehnenirritationen bzw. Rupturen werden durch Implantate bei der dorsalen Plattenosteosynthese selbst oder durch dorsal überstehende Schraubenelemente bei der palmaren Plattenosteosynthese verursacht. Diese werden durch Implantatkorrektur, ME und bedarfsweise durch Naht oder Plastik behandelt. Verhindert werden können diese Komplikationen durch die völlig ausreichende monokortikale Verankerung der von palmar eingebrachten Plattenelemente $[6,13,14]$.

- Beugesehnenläsionen (z.B. Flexor pollicis longus) können durch die palmare Plattenlage begünstigt werden. Verhindert werden kann dies, neben der korrekten mittigen Plattenlage und der Verwendung von Implantaten mit geeignetem Schrauben- und Plattendesign, auch durch die häufig mögliche [11] und immer häufiger geübte Refixation des M. pronator quadratus.

- Zur Vermeidung eines postoperativen Karpaltunnelsyndroms sollte für die palmare Plattenosteosynthese der radiopalmare Zugang ohne Freilegung des N. medianus gewählt werden. Eine regelhafte Karpaltunnelspaltung ist nicht indiziert $[6,12]$.

- Das komplexe regionale Schmerzsyndrom (CRPS) tritt häufiger nach mehrfachen Repositionsmanövern, Nervenirritationen und bei fortbestehender schmerzhafter Fehlstellung sowie einer längeren Immobilisierung auf. Als beste Prophylaxe gilt die schonende schmerzfreie Reposition und übungsstabile operative Retention mit frühfunktioneller Beübung [6,14].

\section{Begleitverletzung}

Am Ende jedes operativen Eingriffs ist die kinematografische Untersuchung unter BV zum Ausschluss von stabilitätsgefährdenden Begleitverletzungen obligat [14]. Zwei Drittel aller distalen Radiusfrakturen zeigen karpale, radioulnare und ulnokarpale Begleitverletzungen, wobei lediglich die Hälfte eine klinische Relevanz besitzt.

Am Ende jedes operativen Eingriffs ist die kinematografische Untersuchung unter AV zum Ausschluss von stabilitätsgefährdenden Begleitverletzungen (z. B. SL-Dissoziation) obligat.

- Nur bei einer relevanten ulnaren Instabilität können refixationsfähige, basisnahe Abbrüche des Processus styloideus ulnae mit KD oder einer kleinen Zuggurtung refixiert und 4 Wochen ruhig gestellt werden [10].

- Bei Läsionen des intrinsischen Bandapparats sollte die statische Instabilität der skapholunären (SL) und lunotriquetralen (LT) Bänder durch offene Naht bzw. Refixation und eine temporäre KD-Transfixation behandelt werden. Die zusätzliche Ruhigstellung im Unterarmgips sollte 6-8 Wochen andauern [9].

- Selten sind distale Radiusfrakturen mit Skaphoidfrakturen kombiniert. Hier finden dann je nach Frakturtyp vorzugsweise offene und minimalinvasive Osteosyntheseverfahren zur Sicherstellung einer möglichst frühzeitigen Beübung Anwendung. Eine initial einsetzende Medianussymptomatik muss neben einer sofortigen Frakturversorgung über einen palmaren $\mathrm{Zu}$ gang auch eine Neurolyse des N. medianus und Karpaltunnelspaltung beinhalten.

\section{Fazit}

Mit der deutlich rückläufigen Toleranz von Fehlstellungen und der Verwendung winkelstabiler Implantate werden zunehmend bessere Ergebnisse nach operativer Behandlung der distalen Radiusfraktur beschrieben. Dabei gilt der anatomischen Reposition und übungsstabilen Retention alle Aufmerksamkeit. Dorsale wie v.a. palmare Plattenosteosynthesen ermöglichen dieses Vorgehen. Gelenkstufen über $1 \mathrm{~mm}$, ein Ulnavorschub von über $2 \mathrm{~mm}$ und eine Verkippung der Radiusbasis um mehr als $10^{\circ}$ stellen Präarthrosen dar und sollten heute nicht mehr toleriert werden.

\section{Literatur}

${ }^{1}$ Figl M, Weninger P, Liska $M$ et al. Volar fixedangle plate osteosynthesis of unstable distal radius fractures: 12 months results. Arch Orthop Trauma Surg 2009; 129: 661-669
${ }^{2}$ Gradl G, Wendt M, Gierer P et al. Die Versorgung distaler Radiusfrakturen mit dem Targon $\mathrm{DR}^{\circledR}$-Nagel. Oper Orthop Traumatol 2009; 21: 472-483

${ }^{3}$ Haus BM, Jupiter JB. Intra-articular fractures of the distal end of the radius in young adults: reexamined as evidence-based and outcomes medicine. J Bone Joint Surg [Am] 2009; 91: 2984-2991

${ }^{4} \mathrm{Hotz}$ TK. Osteosynthese distaler Radiusfrakturen mit der Kirschner-Draht-Osteosynthese. OP-Journal 2003; 19: 21-27

${ }^{5}$ Klonz A, Reilmann H. Palmare Plattenosteosynthese der distalen Radiusfraktur. Unfallchirurg 2005; 108: 155-157

${ }^{6}$ Pabst T, Uzdil T, Winker KH. Die palmare Plattenosteosynthese bei distaler Radiusfraktur: Indikation und Technik. OP-Journal 2003, 19: 42-50

7 Peine R, Rikli DA et al. Comparison of three different plating techniques for the dorsum of the distal radius: Biomechanical study. J Hand Surg [Am] 2000; 25: 29-33

8 Rikli DA, Regazzoni T. The double plating technique for distal radius fractures. Tech Hand Upper Ext Surg 2000; 4: 107-114

9 Siebert HR, Klonz A. Distale Radiusfraktur. Unfallchirurg 2005; 108: 135-153

${ }^{10}$ Souer JS, Ring D, Matschke S et al.; AOCID Prospective ORIF Distal Radius Study Group. Effect of an unrepaired fracture of the ulnar styloid base on outcome after plate-and-screw fixation of a distal radial fracture. J Bone Joint Surg [Am] 2009; 91: 830-838

11 Swigart CR, Badon MA, Bruegel VL et al. Assessment of pronator quadratus repair integrity following volar plate fixation for distal radius fractures: a prospective clinical cohort study. J Hand Surg [Am] 2012; 37: 18681873

12 Uzdil T, Neumann W, Bauschke A et al. Die palmare winkelstabile Plattenosteosynthese bei distalen Radiusextensionsfrakturen. Akt Traumatol 2001; 31: 141-148

${ }^{13}$ Winker $K H$. Winkelstabile Plattensysteme am distalen Radius: Lösungsmöglichkeiten und Probleme. Akt Traumatol 2005; 35: 151-154

${ }^{14}$ Winker KH, Uzdil T. Distale Radiusfrakturen. In: Winker KH, Hrsg. Orthopädie-Unfallchirurgie. München: Elsevier; 2011: 596-614

\section{Prof. Dr. med. Karl Heinrich Winker} Chefarzt

Klinik für Unfallchirurgie

und Orthopädie

HELIOS-Klinikum

Nordhäuser Straße 74

99089 Erfurt

karl-heinrich.winker@

helios-kliniken.de

\section{PD Dr. med. Daniel Rikli}

Leitender Arzt Traumatologie

Universitätsspital

Spitalstrasse 21

4031 Basel

Schweiz

drikli@uhbs.ch 